\title{
Distinct Modes of Neuron Addition in Adult Mouse Neurogenesis
}

\author{
Jovica Ninkovic, ${ }^{1}$ Tetsuji Mori, ${ }^{1}$ and Magdalena Götz ${ }^{1,2}$ \\ ${ }^{1}$ Institute for Stem Cell Research, Gesellschaft für Strahlenforschung-National Research Institute for Environment and Health, 85764 Neuherberg/Munich, \\ Germany, and 2Physiological Genomics, University of Munich, 80633 Munich, Germany
}

\begin{abstract}
Adult neurogenesis is restricted to two distinct areas of the mammalian brain: the olfactory bulb (OB) and the dentate gyrus (DG). Despite its spatial restriction, adult neurogenesis is of crucial importance for sensory processing and learning and memory. Although it has been shown that tens of thousands of new neurons arrive in the OB and DG every day with about half of them surviving after integration, the total contribution of adult neurogenesis to the pre-existing network remains mostly unknown. This is because of previous approaches labeling only a small proportion of adult-generated neurons. Here, we used genetic fate mapping to follow the majority of adult-generated neurons over long periods. Our data demonstrate two distinct modes of neuron addition to the pre-existing network. In the glomerular layer of the $\mathrm{OB}$, there is a constant net addition of adult-generated neurons reaching a third of the total neuronal population within 9 months. In contrast, adult neurogenesis contributes to only a minor fraction of the entire neuronal network in the granular cell layer of the $\mathrm{OB}$ and the DG. Although the fraction of adult generated neurons can be further increased by an enriched environment, it still remains a minority of the neuronal network in the DG. Thus, neuron addition is distinct and tightly regulated in the neuronal networks that incorporate new neurons life long.
\end{abstract}

Key words: granule cells; periglomerular neurons; dentate gyrus; olfactory bulb; neuron integration; neural network

\section{Introduction}

Neurogenesis continues into adulthood in two areas of the mammalian brain (Ming and Song, 2005). Neural stem or progenitor cells located in the subgranular zone (SGZ) generate glutamatergic neurons that play an important role for certain forms of learning, memory and depression (Nilsson et al., 1999; Dranovsky and Hen, 2006). In the subependymal zone (SEZ), neural stem cells generate mainly GABAergic interneurons that migrate mostly into the granular cell layer (GCL) of the olfactory bulb (OB) (Petreanu and Alvarez-Buylla, 2002; Belluzzi et al., 2003; Saghatelyan et al., 2003; Lemasson et al., 2005; Lledo and Saghatelyan, 2005). Previous evidence suggests that many of the adultgenerated neurons settling in the GL of the $\mathrm{OB}$ also originate from a further dorsorostral position in the rostral migratory stream (RMS) (Hack et al., 2005; Merkle et al., 2007). Adultgenerated neurons in the OB play an important role for odor discrimination (Nilsson et al., 1999; Rochefort et al., 2002; Dranovsky and Hen, 2006; Gheusi and Lledo, 2007). Despite its prominent functional importance, many aspects of adult neuro-

Received June 6, 2007; revised Aug. 7, 2007; accepted Aug. 15, 2007.

This work was supported by the Deutsche Forschungsgemeinschaft, Bundesministerium fur Bildung und Forschung, and the European Union. We are very grateful to Pierre-Marie Lledo and Adi Mizrahi for helpful discussions, to Leanne Godinho and Chichung Lie for excellent suggestions on this manuscript, and to Andrea Steiner-Mezzadri and Anette Jäckle for technical assistance.

Correspondence should be addressed to Magdalena Götz at the above address. E-mail: magdalena.goetz@gsf.de.

T. Mori's present address: Department of Anatomy and Cell Science, Kansai Medical University, 10 -15 Fumizonocho, Moriguchi, Osaka 570-8506, Japan.

DOI:10.1523/JNEUROSCI.2572-07.2007

Copyright $\odot 2007$ Society for Neuroscience ～0270-6474/07/2710906-06\$15.00/0 genesis, such as the total turnover rate of adult-generated neurons and their contribution to the existing network still remain mostly unknown. This is because, thus far, it has only been possible to label a single cohort of adult-generated neurons.

Previous approaches either used administration of DNA base analogues (Kuhn et al., 1996; Cameron and McKay, 2001; Petreanu and Alvarez-Buylla, 2002; Kempermann et al., 2003) or infection with retroviral vectors (Petreanu and Alvarez-Buylla, 2002; van Praag et al., 2002; Zhao et al., 2006). These studies revealed that between 10 and 30,000 cells are generated per day (Alvarez-Buylla et al., 2001; Cameron and McKay, 2001) and that about half of these die within the first 4 weeks (Petreanu and Alvarez-Buylla, 2002; Kempermann et al., 2003; Lemasson et al., 2005). Whereas some studies suggested that most cells that survived initial 4 weeks could still be detected after several months (Kempermann et al., 2003; Lemasson et al., 2005), others reported a further decrease in the number of labeled neurons during $365 \mathrm{~d}$ of survival (Petreanu and Alvarez-Buylla, 2002; Kohwi et al., 2007). Extrapolating these data from labeling a single or few cohorts would suggest a considerable contribution of adultgenerated neurons to the OB network, leading several studies to propose an increase in the size of the dentate gyrus (DG) and the $\mathrm{OB}$ attributable to adult neurogenesis (Kempermann et al., 2003; Merson et al., 2006). Although the majority of the BrdU analysis focused on the GCL, in vivo imaging could only access the GL where a turnover of only $3 \%$ of the neurons was observed (Mizrahi et al., 2006). Thus, a certain degree of inconsistency between different reports may be attributable to the relatively short time frame analyzed by imaging or the restriction to labeling few cohorts of adult-generated cells. These limitations may 
explain why the indirect extrapolation of the above data leads to diverse predictions of the number of the entire population of adult-generated neurons. To overcome these limitations, we used permanent genetic fate mapping to monitor the majority of adult-generated neurons over 9 months.

\section{Materials and Methods}

Animals. GLAST::CreERT2 mice (Mori et al., 2006) were crossed to R26R or Z/EG maintained on a C57BL6/J background. Animals were group housed in polypropylene cages lined with wood chips, if not stated otherwise. A running wheel and two $12-\mathrm{cm}$-long tubes were added to provide an enriched environment (Kempermann et al., 1998; van Praag et al., 1999). All experimental procedures were performed in accordance with German and European Union guidelines.

Tamoxifen administration and adult neurogenesis monitoring. Tamoxifen (TM; T-5648; Sigma, Munich, Germany) was dissolved at $20 \mathrm{mg} / \mathrm{ml}$ in corn oil (C-8267; Sigma) and 3-month-old animals were intraperitoneally injected with $1 \mathrm{mg}$ twice a day for 5 consecutive days (Mori et al., 2006). To control for a potential loss of reporter activity, we quantified labeled cells in the cerebral cortex and other parenchymal regions without any neuronal turnover and observed no difference in the number of labeled cells. Moreover, R26R as well as Z/EG mice showed labeling in all DG and OB neurons when crossed to Cre-lines that mediate recombination at embryonic stages (Tang et al., 2002; Malatesta et al., 2003). We observed no alterations in adult neurogenesis between WT and GLAST::CreERT2 heterozygous mice, consistent with previous work reporting the lack of a phenotype in mice heterozygous for the astrocytespecific glutamate transporter (GLAST) (Voutsinos-Porche et al., 2003).

BrdU treatment. The DNA base analog 5-bromodeoxyuridine (BrdU, B-5002; Sigma) (100 mg/kg body weight) was either injected intraperitoneally three times with $12 \mathrm{~h}$ intervals starting at $36 \mathrm{~h}$ before perfusion (supplemental Fig. 3, available at www.jneurosci.org as supplemental material) or BrdU was given in the drinking water $(1 \mathrm{mg} / \mathrm{ml})$ for 2 weeks followed by 2 weeks normal water (supplemental Fig. 1, available at www.jneurosci.org as supplemental material).

Histology and immunostaining. Adult mice were transcardially perfused with $4 \%$ paraformaldehyde (PFA) in PBS. After postfixation and cryoprotection, $30-\mu \mathrm{m}$-thick sections were cut at the cryostat and stained with the following antibodies diluted in PBS containing $10 \%$ normal goat serum and $0.5 \%$ Triton X-100: anti-NeuN (1:75; Millipore, Temecula, CA), anti-GFAP (1:100; Sigma), anti-doublecortin (DCX; 1:1000; Millipore), anti-calbindin (1: 200; Swant, Bellinzona, Switzerland), anti-calretinin (1:200; Swant), anti-TH (1:200; Millipore), and anti- $\beta$-galactosidase (1:5000; Cappel, Cochranville, PA). The tyramide amplification system (PerkinElmer, Wellesley, MA) was used to detect $\beta$-galactosidase-immunoreactivity according to the manufacturer's protocol. BrdU was detected using anti-BrdU antibody (1:200, Abcam, Cambridge, UK) after $2 \mathrm{M} \mathrm{HCl}$ pretreatment. Appropriate secondary antibodies conjugated to Cy2 or Cy3 (1:200, Jackson Laboratory) were applied to detect the primary antibody. The images were taken using an Olympus FV1000 confocal microscope. Sections were optically sectioned using $1 \mu \mathrm{m}$ Z-intervals, and cells were rotated in orthogonal planes to verify double labeling.

Quantitative analysis. Quantifications (proportions of reporter+ cells among particular cell types) were done using ImageJ software to analyze confocal Z-stacks. Nine sections sampled every $90 \mu \mathrm{m}$ from the midline were analyzed per animal and for every time point three animals were analyzed. All results are presented as mean and SEM unless indicated otherwise. Statistical analysis was performed using Microcal Origin 7.5.

\section{Results}

Here, we aimed to monitor the entire population of adultgenerated neurons by TM-induced recombination in adult neural stem cells in a mouse line expressing TM-inducible form of Cre recombinase (CreERT2) in the locus of GLAST (Mori et al., 2006). The progeny of cells that underwent recombination was followed by expression of a reporter gene (either $\beta$-galactosidase in $R 26 R$ or GFP in $Z / E G$ ) (Mori et al., 2006) for up to 9 months after induction (Fig. 1a) and no downregulation of reporter activity was observed (Fig. 1d) (see Material and Methods). After TM application in 3-month-old GLAST::CreERT2/R26R mice, $\beta$-galactosidase-immunoreactivity was observed in a considerable proportion of GFAP+ astrocytes as early as $5 \mathrm{~d}$ in the adult SEZ and the SGZ of the DG (Fig. 1b,c) (Mori et al., 2006). A label-retaining protocol (see Materials and Methods) also detected slow-dividing stem cells among the reporter + GFAP+ cells. Notably, reporter + cells constituted the majority of all BrdU-retaining stem cells for up to 9 months after TM application (supplemental Fig. $1 a-c$, available at www.jneurosci.org as supplemental material). Consistent with this efficient recombination in stem cells, the proportion of reporter + newly generated neurons labeled by DCX reached a stable proportion of over $90 \%$ for up to 9 months (Fig. 1d,e). These data therefore demonstrate a very efficient labeling of stem cells in the adult neurogenic zones that continue to generate the majority of new neurons over a long time.

Although shortly (5 d) after TM application no mature $\mathrm{NeuN}+$ neurons were yet reporter $+(\beta$-galactosidase + or GFP + ) (data not shown), double-positive neurons were present in the granule cell layer (GCL) and glomerular layer (GL) of the OB (Fig. 2) and the DG (Fig. 3) after 1.5 months. The newly generated neurons integrate mainly in the lower part of the upper DG blade (Fig. $3 a$, supplemental Fig. 3 , available at www.jneurosci.org as supplemental material), but uniformly in the OB (Fig. $2 a-d$ ). Similar data were obtained with $R 26 R$ (Figs. 2, 3) and $Z / E G$ reporter mice. Despite careful examination of the entire forebrain, no addition of DCX + or NeuN + and reporter + neurons could be detected outside the DG and OB (data not shown). Within the GL of the OB, we noted a considerable increase of reporter+ neurons between 1.5 and 9 months after TM (Fig. $2 a, b)$. Quantification of the proportion of reporter + (i.e., adultborn) neurons at the different stages after TM application revealed a constant (linear) increase reaching 32\% of all periglomerular neurons (PGNs) 9 months after TM induction (Fig. 2e). Neurons in the GL are a heterogeneous population comprising GABAergic neurons (55\%) that also comprise the dopaminergic and the calbindin + interneurons. Conversely, the majority of calretinin + PGNs appear not to be GABAergic (Kosaka et al., 1995; Parrish-Aungst et al., 2007). Among these populations, only dopaminergic and calretinin + neurons were reporter+ (supplemental Fig. 2, available at www.jneurosci.org as supplemental material). Consistent with the predominantly embryonic origin of calbindin+ neurons (De Marchis et al., 2007) (but see Kohwi et al., 2007), this subset was consistently $\beta$-galactosidasenegative (supplemental Fig. $2 a$, available at www.jneurosci.org as supplemental material). Conversely, $\sim 40 \%$ of all $\beta$-galactosidase + PGNs were immunopositive for tyrosine hydroxylase (TH) (Fig. $2 f$, supplemental Fig. $2 b$, available at www.jneurosci.org as supplemental material), consistent with their generation throughout adulthood (Hack et al., 2005). The other major subtype of adult generated PGNs was the calretinin + population that reached $\sim 50 \%$ of all $\beta$-galactosidase + PGNs 6 months after induction (Fig. $2 f$, supplemental Fig. $2 c$, available at www.jneurosci.org as supplemental material). However, there is also a small population of $\beta$-galactosidase + neurons in the GL that remain yet uncharacterized. Thus, mainly two subtypes of PGNs are added from the GLAST + progenitors during adult neurogenesis at a slightly different pace (Fig. 2f). Interestingly, the increase in both of these subtypes followed the increase in reporter+ neurons labeled by NeuN. Quantification of the proportion of reporter $+\mathrm{TH}+$ neurons among all $\mathrm{TH}+$ neurons 
over time also demonstrated a linear increase in the reporter + fraction reaching $23.7 \pm 3.2 \%$ (three animals) after 9 months. Thus, these data demonstrate a constant net addition of two subtypes of adult generated PGNs to the total neuronal population in the GL.

Conversely, we observed a rather different mode of neuron addition in the GCL of the OB (Fig. $2 c-e$ ). Although the proportion of adult-generated reporter+ neurons among all GCL neurons increased to $13.8 \%$ within the first 4 months after TM induction (Fig. 2e), surprisingly, no further increase was observed thereafter (Fig. 2e) ( $p=0.4$, independent $t$ test, cross-comparison of 4,6 , and 9 months of survival). A strikingly similar pattern to the one observed in the GCL was detected in the DG (Fig. $3 a-c$ ) where the proportion of reporter + neurons among all $\mathrm{NeuN}+$ cells also reached a plateau at $\sim 4$ months after TM application (Fig. 2c) ( $p \leq 0.96$, independent $t$ test, crosscomparison of 4,6 , and 9 months survival). The only change observed between 4 and 9 months after TM application was a gradual redistribution of a small proportion of $\beta$-galactosidase + neurons into the upper half of the granule layer in the DG $(<20 \%$ of all adult-generated neurons (supplemental Fig. $3 a-c$, available at www.jneurosci.org as supplemental material), whereas no obvious changes in the more wide-spread distribution were observed in the GCL of the OB (Fig. $2 c, d$ ). Thus, adult-generated neurons seemingly constitute only a minor proportion of the total neurons in the DG and GCL of the OB. Notably, most adult-generated neurons remained localized in the lower half of the granule cell layer in the DG, but no specific distribution could be detected in the GCL of the OB.

To examine whether the consequence of previously described physiological stimuli acting on neurogenesis and neuronal incorporation (Kempermann et al., 1998; van Praag et al., 1999) can be detected by our fate-mapping technique, we examined the effect of an enriched environment on the contribution of reporter + neurons to the DG neuronal population. Consistent with previous data, exposure of mice to an enriched environment for only 2 weeks resulted in a significant increase of cell division (supplemental Fig. $4 a-c$, available at www.jneurosci.org as supplemental material). When littermate GLAST::CreERT2 mice were housed from 7 to 9 months after TM in an enriched environment, this resulted in an additional increase (by $67 \%$ ) in the proportion of $\beta$-galactosidase + neurons among all DG neurons compared with their littermates housed in normal cages (Fig. $3 c-e)(p=0.018)$. This increase occurred mostly in the deep layers closer to the SGZ in the DG (Fig. $3 d$,e), consistent with the addition of newly generated neurons resulting from exercise-stimulated neurogenesis. Moreover, enriched lower blade in the DG.
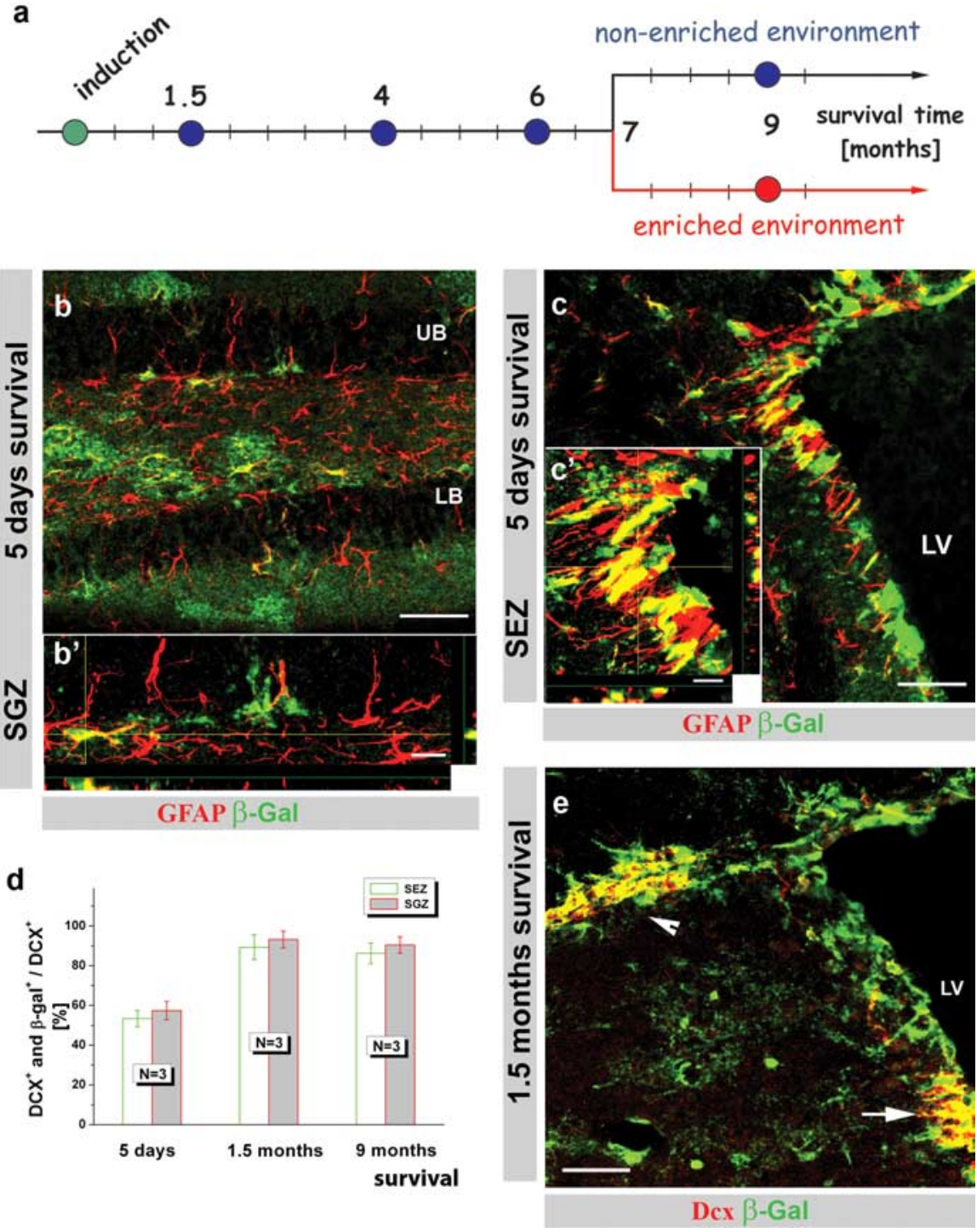

Figure 1. GLAST::CreERT2 knock-in mice are a useful tool to study adult neurogenesis. $\boldsymbol{a}$, Schematic drawing of the experimentime points thereafter. $\boldsymbol{b}, \boldsymbol{c}$, Micrographs depicting immunostaining for $\beta$-galactosidase reporter activity (green) and GFAP (red) in the SGZ of the DG $(\boldsymbol{b}, \boldsymbol{b})$ and SEZ $(\boldsymbol{c}, \boldsymbol{c})$. The histograms in $\boldsymbol{d}$ depict the quantification of reporter + neuroblasts $(D C X+)$ among ing $\beta$-galactosidase + (green) neuroblasts labeled for DCX (red) 9 months after TM induction in the SEZ (white arrow) and the

housing conditions also resulted in an increase in reporter + neuron addition in the lower blade of the DG (Fig. $3 d, e$, supplemental Fig. $4 d$, available at www.jneurosci.org as supplemental material) that remained, however, at lower levels compared with neurogenesis in the upper blade (supplemental Fig. $4 d$, available at www.jneurosci.org as supplemental material). Thus, the limited contribution of adult-generated neurons to the entire neuronal population in the DG reflects an actively regulated level rather than a technical short-coming of our labeling.

\section{Discussion}

Together, our genetic fate-mapping analysis suggests the existence of two modes of adult neurogenesis and its contribution to the existing neuronal network. In the GL of the OB, the proportion of newly generated neurons increases continuously because of the net addition of inhibitory interneurons. In contrast, in the 

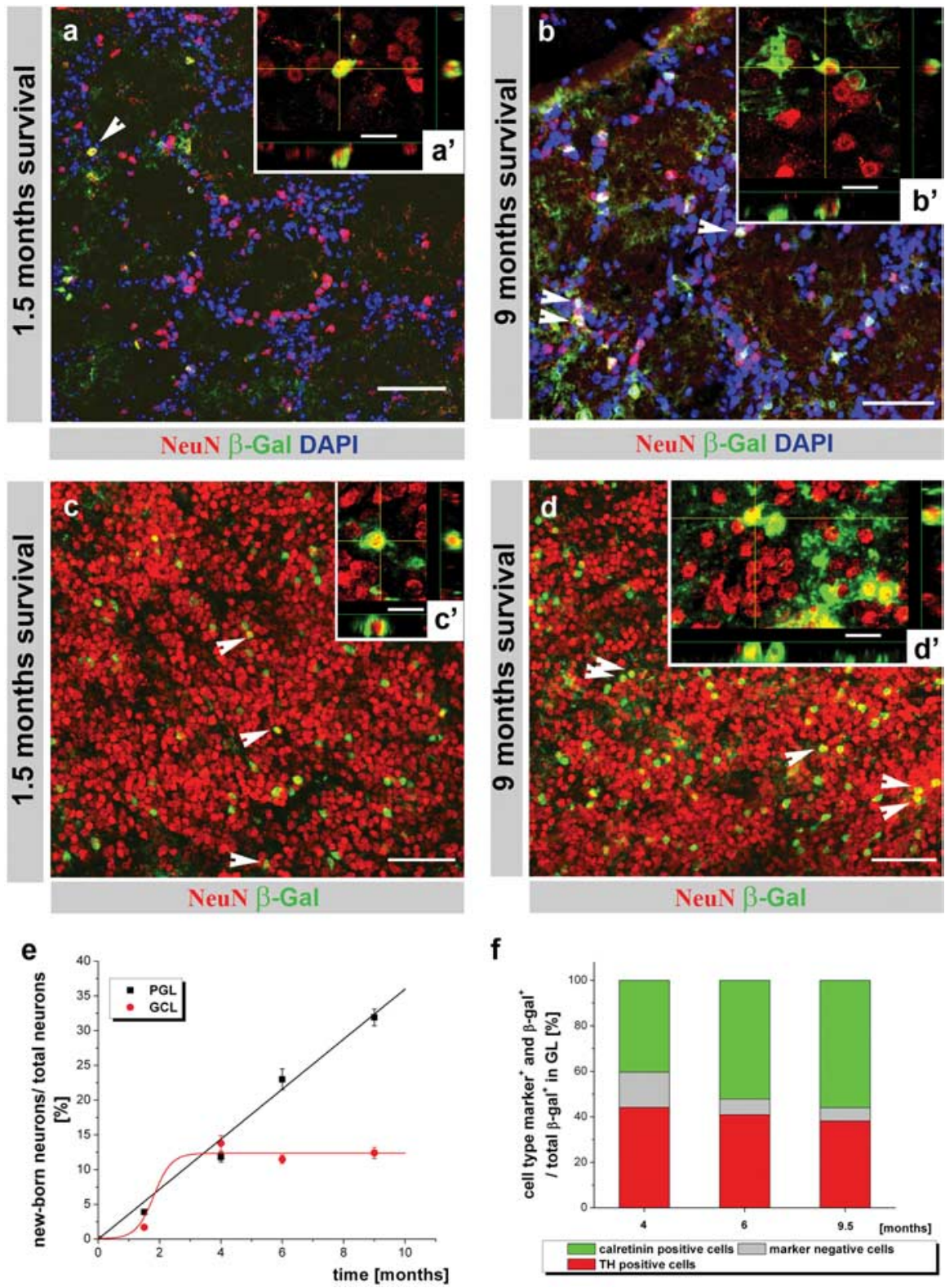

f

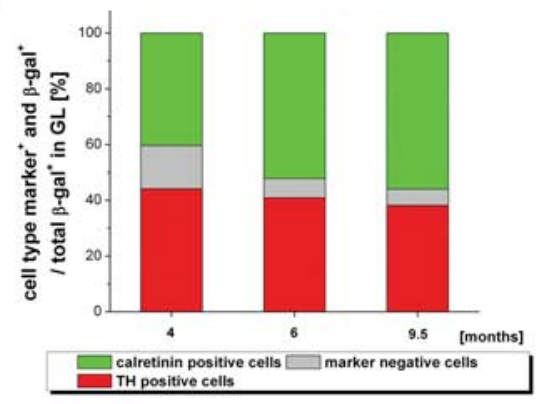

Figure 2. Monitoring adult neurogenesis for 9 months revealed two distinct modes of neuron addition in the OB. $\boldsymbol{a}-\boldsymbol{d}$, Fluorescence micrographs depicting NeuN + (red) neurons double-labeled for the $\beta$-galactosidase reporter (green, white arrowheads) 1.5 months $(\boldsymbol{a}, \boldsymbol{c})$ and 9 months $(\boldsymbol{b}, \boldsymbol{d})$ after TM application in the $\mathrm{GL}(\boldsymbol{a}, \boldsymbol{b})$ and the $\mathrm{GCL}(\boldsymbol{c}, \boldsymbol{d})$ of the OB. Scale bars: $\boldsymbol{b}, \boldsymbol{c}, \boldsymbol{e}-\boldsymbol{g}$, $50 \mu \mathrm{m} ; \boldsymbol{b}^{\prime}, \boldsymbol{c}^{\prime}, \boldsymbol{e}^{\prime}-\boldsymbol{g}^{\prime}, 10 \mu \mathrm{m}$. LV, Lateral ventricle; UB, upper blade, LB, lower blade in the DG. Higher-power insets represent 10 $\mu \mathrm{m} z$-stack with $x-z$ and $y-z$ projections. $\boldsymbol{e}$, Histograms depicting the mean ( \pm SEM) proportion of recombined, $\beta$-galactosidase + neurons among all neurons analyzed in three animals for each time point in the $\mathrm{GL}$ and $\mathrm{GCL}$. The calculations of the best fit curve are $y=a x$, where $a=3.6$ for GL and $y=A_{1}+\left(A_{2}-A_{1}\right) /\left(1+10^{\log \left(x_{0}-x\right) p}\right)$, where $A_{1}=0.07, A_{2}=12.4$, $\log x_{0}=1.8$, and $p=1.5$ for GCL. Note that labeled neurons constantly increase in the $\mathrm{GL}$, although they remain at a constant, minor proportion in the $\mathrm{GCL}$ of the $0 \mathrm{~B}$. $\boldsymbol{f}$, Histogram of the proportion of neuronal subtypes among all $\beta$-galactosidase + cells in the GL. The fraction of neuronal subtypes is shown as a mean for three animals for every time point. Scale bars: $\boldsymbol{a}, \boldsymbol{c}, \boldsymbol{d}, 50 \mu \mathrm{m} ; \boldsymbol{b}$, $40 \mu \mathrm{m} ; \boldsymbol{a}^{\prime}-\boldsymbol{d}^{\prime}, 10 \mu \mathrm{m}$.

DG and GCL of the OB, the proportion of newly generated neurons reaches a plateau with a relatively minor contribution to the entire population of neurons that remains stable at this low proportion for many months. These results are not attributable to technical limitations of reporter activity, as we still detect $90 \%$ of all newly generated neurons in the DG and OB being reporter+. Moreover, the proportion of slow-dividing stem cells that was reporter + remained stable over many months. Finally, report- er+ neurons further increase after a known stimulus of adult neurogenesis, the enriched environment with access to a running wheel. Thus, newly generated neurons are efficiently and stably labeled. We therefore conclude that the addition of reporter + neurons to the pre-existing neurons is regulated rather differently in the DG and the GCL of the OB compared with the GL of the OB.

The findings obtained by directly monitoring the bulk of adult-generated neurons reconcile different outcomes of previous studies looking at smaller numbers of neurons by different techniques. For example, the low degree of disappearance of pre-existing neurons imaged in the GL (Mizrahi et al., 2006) is consistent with the net addition of newly arriving neurons there implying no or little cell death of previously generated neurons. Moreover, the transgenic GFP-labeling used in this study primarily targets calbindin + neurons that we find not to be generated in the adult at least as detected by the GLAST::CreERT2-mediated fate-mapping approach labeling $90 \%$ of all newly generated neurons. Our data are also in line with the prediction of a net addition of neurons in the GL, as suggested by previous BrdU labeling (Petreanu and Alvarez-Buylla, 2002). However, this previous work using ${ }^{3} \mathrm{H}$ Thymidin or BrdU labeling of few cohorts of adult generated neurons would predict a considerably larger amount of neuron addition ranging from 50 to $80 \%$, depending on the assumption on the cell cycle length (supplemental text, available at www.jneurosci. org as supplemental material). Finally, none of the previous publications had ever suggested a different mode of neuron addition in the GCL versus the GL. Thus, the use of genetic fate mapping provides new insights into the total population of adult generated neurons, both their identity (only dopaminergic and calretinin + in the GL) and their numbers.

A striking result of our fate-mapping analysis is not only the difference in the mode of neuron addition between the GL and the GCL/DG, but also the relatively low contribution of adult-generated neurons to the neuron pool in the DG or GCL in the OB barely exceeding $14 \%$ of the total neuronal population. This proportion remains low even after potent physiological stimuli. Thus, despite the daily arrival of several thousands of new neurons their contribution to the total neuron pool is rather limited and does not change significantly with age despite the decrease in proliferation in both SEZ and SGZ with age (Kuhn et al., 1996). These data thus imply an equilibrium between dying and integrating adult generated neurons that is regulated according todemand. By these means the neuronal network contains a small pool of plastic neu- 
rons, many of which are constantly renewed within a larger proportion of neurons that have been generated at earlier stages and are not turned over. Indeed, induction of recombination by TM shortly after birth [postnatal day (P0)] labels notably higher proportions of neurons in the $\mathrm{OB}$ compared with the same survival stage after TM application in 3-month-old mice (percentage of newly generated neurons from $\mathrm{P} 0,8.7 \pm 0.6 \%$ in the GL and $13.1 \pm 1.1 \%$ in the GCL; $<5 \%$ after TM at 3 months) (Fig. $2 e$ ). Together, these data suggest that neurons generated at early stages represent a rather stable pool, thereby providing the network stability requested at the theoretical level, while still allowing plasticity (SchmidtHieber et al., 2004). Indeed, a continuous addition of excitatory neurons to the DG would result in overexcitation and epileptogenesis, which is avoided by a continuous turnover of the newly added neurons (Meltzer et al., 2005). Strikingly, the converse pattern of neuron addition with a constant increase in the contribution of adult generated neurons to the network occurs exactly in the region of the $\mathrm{OB}$ that first modulates the input from the olfactory neurons. Olfactory neurons are constantly replaced and newly generated (Lledo et al., 2004), prompting the speculation that these two events are correlated. A continuous new population of afferents may not require stable network components, but rather take advantage of a constant adaptivity of sensory relay. Conversely, memory processing in the DG may rely on a good proportion of stable parts of the network. These hypotheses highlight the importance of our data for additional work on neural network function in these systems, helping to elucidate the contribution of adult neurogenesis to neuronal information processing.

\section{References}

Alvarez-Buylla A, Garcia-Verdugo JM, Tramontin AD (2001) A unified hypothesis on the lineage of neural stem cells. Nat Rev Neurosci 2:287-293.

Belluzzi O, Benedusi M, Ackman J, LoTurco JJ (2003) Electrophysiological differentiation of new neurons in the olfactory bulb. J Neurosci 23:10411-10418.

Cameron HA, McKay RD (2001) Adult neurogenesis produces a large pool of new granule cells in the dentate gyrus. J Comp Neurol 435:406-417.

De Marchis S, Bovetti S, Carletti B, Hsieh YC, Garzotto D, Peretto P, Fasolo A, Puche AC, Rossi F (2007) Generation of distinct types of periglomerular olfactory bulb interneurons during development and in adult mice: implication for intrinsic properties of the subventricular zone progenitor population. J Neurosci 27:657-664.

Dranovsky A, Hen R (2006) Hippocampal neurogenesis: regulation by stress and antidepressants. Biol Psychiatry 59:1136-1143.

Gheusi G, Lledo PM (2007) Control of early events in olfactory processing by adult neurogenesis. Chem Senses 32:397-409.

Hack MA, Saghatelyan A, de Chevigny A, Pfeifer A, Ashery-Padan R, Lledo PM, Gotz M (2005) Neuronal fate determinants of adult olfactory bulb neurogenesis. Nat Neurosci 8:865-872.

Kempermann G, Kuhn HG, Gage FH (1998) Experience-induced neurogenesis in the senescent dentate gyrus. J Neurosci 18:3206-3212.
Kempermann G, Gast D, Kronenberg G, Yamaguchi M, Gage FH (2003) Early determination and long-term persistence of adult-generated new neurons in the hippocampus of mice. Development 130:391-399.

Kohwi M, Petryniak MA, Long JE, Ekker M, Obata K, Yanagawa Y, Rubenstein JLR, Alvarez-Buylla A (2007) A subpopulation of olfactory bulb GABAergic interneurons is derived from Emx1- and Dlx5/6-expressing progenitors. J Neurosci 26:6878-6891.

Kosaka K, Aika Y, Toida K, Heizmann CW, Hunziker W, Jacobowitz DM, Nagatsu I, Streit P, Visser TJ, Kosaka T (1995) Chemically defined neuron groups and their subpopulations in the glomerular layer of the rat main olfactory bulb. Neurosci Res 23:73-88.

Kuhn HG, Dickinson-Anson H, Gage FH (1996) Neurogenesis in the dentate gyrus of the adult rat: age-related decrease of neuronal progenitor proliferation. J Neurosci 16:2027-2033.

Lemasson M, Saghatelyan A, Olivo-Marin JC, Lledo PM (2005) Neonatal and adult neurogenesis provide two distinct populations of newborn neurons to the mouse olfactory bulb. J Neurosci 25:6816-6825.

Lledo PM, Saghatelyan A (2005) Integrating new neurons into the adult olfactory bulb: joining the network, life-death decisions, and the effects of sensory experience. Trends Neurosci 28:248-254.

Lledo PM, Saghatelyan A, Lemasson M (2004) Inhibitory interneurons in the olfactory bulb: from development to function. Neuroscientist 10:292-303. 
Malatesta P, Hack MA, Hartfuss E, Kettenmann H, Klinkert W, Kirchhoff F, Gotz M (2003) Neuronal or glial progeny: regional differences in radial glia fate. Neuron 37:751-764.

Meltzer LA, Yabaluri R, Deisseroth K (2005) A role for circuit homeostasis in adult neurogenesis. Trends Neurosci 28:653-660.

Merkle FT, Mirzadeh Z, Alvarez-Buylla (2007) Mosaic organization of neural stem cells in the adult brain. Science 317:381-384.

Merson TD, Dixon MP, Collin C, Rietze RL, Bartlett PF, Thomas T, Voss AK (2006) The transcriptional coactivator Querkopf controls adult neurogenesis. J Neurosci 26:11359-11370.

Ming GL, Song H (2005) Adult neurogenesis in the mammalian central nervous system. Annu Rev Neurosci 28:223-250.

Mizrahi A, Lu J, Irving R, Feng G, Katz LC (2006) In vivo imaging of juxtaglomerular neuron turnover in the mouse olfactory bulb. Proc Natl Acad Sci USA 103:1912-1917.

Mori T, Tanaka K, Buffo A, Wurst W, Kuhn R, Gotz M (2006) Inducible gene deletion in astroglia and radial glia - a valuable tool for functional and lineage analysis. Glia 54:21-34.

Nilsson M, Perfilieva E, Johansson U, Orwar O, Eriksson PS (1999) Enriched environment increases neurogenesis in the adult rat dentate gyrus and improves spatial memory. J Neurobiol 39:569-578.

Parrish-Aungst S, Shipley MT, Erdelyi F, Szabo G, Puche AC (2007) Quantitative analysis of neuronal diversity in the mouse olfactory bulb. J Comp Neurol 501:825-836.

Petreanu L, Alvarez-Buylla A (2002) Maturation and death of adult-born olfactory bulb granule neurons: role of olfaction. J Neurosci 22:6106-6113.

Rochefort C, Gheusi G, Vincent JD, Lledo PM (2002) Enriched odor exposure increases the number of newborn neurons in the adult olfactory bulb and improves odor memory. J Neurosci 22:2679-2689.

Saghatelyan A, Carleton A, Lagier S, de Chevigny A, Lledo PM (2003) Local neurons play key roles in the mammalian olfactory bulb. J Physiol (Paris) 97:517-528.

Schmidt-Hieber C, Jonas P, Bischofberger J (2004) Enhanced synaptic plasticity in newly generated granule cells of the adult hippocampus. Nature 429:184-187.

Tang SH, Silva FJ, Tsark WM, Mann JR (2002) A Cre/loxP-deleter transgenic line in mouse strain 129S1/SvImJ. Genesis 32:199-202.

van Praag H, Kempermann G, Gage FH (1999) Running increases cell proliferation and neurogenesis in the adult mouse dentate gyrus. Nat Neurosci 2:266-270.

van Praag H, Schinder AF, Christie BR, Toni N, Palmer TD, Gage FH (2002) Functional neurogenesis in the adult hippocampus. Nature 415:1030-1034.

Voutsinos-Porche B, Knott G, Tanaka K, Quairiaux C, Welker E, Bonvento G (2003) Glial glutamate transporters and maturation of the mouse somatosensory cortex. Cereb Cortex 13:1110-1121.

Zhao C, Teng EM, Summers Jr RG, Ming GL, Gage FH (2006) Distinct morphological stages of dentate granule neuron maturation in the adult mouse hippocampus. J Neurosci 26:3-11. 